\title{
Configurações
}

Revista Ciências Sociais

$14 \mid 2014$

Para além da Governação. Políticas, práticas e discursos de inclusão e promoção da diversidade cultural

\section{Del discurso a los hechos: el Estado mexicano y los pueblos indígenas de México}

Do discurso aos fatos: 0 estado mexicano e os povos indígenas do México

From words to deeds: the Mexican state and the indigenous peoples of Mexico

\section{Andrea Aguillar Edwards}

\section{OpenEdition}

1 Journals

Edición electrónica

URL: http://journals.openedition.org/configuracoes/2256

DOI: 10.4000/configuracoes.2256

ISSN: $2182-7419$

Editor

Centro de Investigação em Ciências Sociais

Edición impresa

Paginación: 39-66

ISBN: 1646-5075

ISSN: 1646-5075

\section{Referencia electrónica}

Andrea Aguillar Edwards, «Del discurso a los hechos: el Estado mexicano y los pueblos indígenas de México », Configurações [En línea], 14 | 2014, Publicado el 27 marzo 2015, consultado el 10 diciembre 2020. URL : http://journals.openedition.org/configuracoes/2256 ; DOI : https://doi.org/10.4000/ configuracoes. 2256

Este documento fue generado automáticamente el 10 diciembre 2020. 


\title{
Del discurso a los hechos: el Estado mexicano y los pueblos indígenas de México
}

\author{
Do discurso aos fatos: 0 estado mexicano e os povos indígenas do México \\ From words to deeds: the Mexican state and the indigenous peoples of Mexico
}

\section{Andrea Aguillar Edwards}

\section{Introducción}

1 En el marco de la creciente preocupación por la multiculturalidad y los procesos de integración de los pueblos, en plena expansión de un sistema globalizado, es importante destacar el hecho de que la diversidad cultural, étnica y social, existe no sólo entre las diversas sociedades, sino al interior de las mismas. En este trabajo se aborda el fenómeno de la multiculturalidad en México, enfocando el caso específico de los pueblos indígenas que cohabitan el territorio nacional y su lucha permanente por hacer valer su cultura y sus derechos. Se parte de una visión general del contexto histórico de la relación del Estado mexicano y su población indígena, como factor de análisis para la comprensión de la situación actual del fenómeno multicultural y la propuesta política y social del gobierno en turno.

2 Es importante señalar que este trabajo forma parte del proyecto Multilevel governance of cultural diversity in a comparative perspective: EU-Latin America, auspiciado por la Comisión Europea, y se enfoca al caso particular de México, sin desconocer el hecho de que otros países latinoamericanos enfrentan una situación similar ${ }^{1}$. Como primer avance, se identifican las estrategias del gobierno federal mexicano enfocadas al desarrollo de los pueblos indígenas, durante los cinco sexenios previos a la administración actual del presidente Enrique Peña Nieto. Se registra el seguimiento del movimiento indígena durante el mismo período, destacando uno de los acontecimientos sociales de mayor trascendencia en México y en la opinión pública internacional: el levantamiento armado 
de los pueblos indígenas en Chiapas, en 1994, como principal referente para el análisis del proceso político-social que precede al establecimiento de las actuales políticas públicas y estrategias del gobierno mexicano.

\section{El tema del multiculturalismo}

3 Para efectos de este trabajo entenderemos por multiculturalismo al reconocimiento de las diferencias sociales, étnicas, culturales, religiosas, y aquellas que definan una característica distintiva de la idiosincrasia de un grupo, en el diseño de un sistema social que garantice la equidad y la armonía de intereses. El multiculturalismo se concibe entonces relacionado con la forma en que los gobiernos administran los recursos y diseñan las políticas públicas. Es decir, para que la multiculturalidad presente en un país, en forma natural, se traduzca en un sistema multiculturalista, debe mediar un proyecto social capaz de insertar a todos, en igualdad de derechos y de oportunidades para ejercerlos.

4 La etnicidad es hoy un factor determinante de los lineamientos que dictan los organismos internacionales de desarrollo para la elaboración de políticas públicas. Entre ellos, los de mayor influencia, como la Organización de las Naciones Unidas ${ }^{2}$ (ONU) y el Banco Mundial ${ }^{3}$ (BM), entre otros, consideran el reconocimiento de la diversidad cultural no solo como un derecho, sino como un requisito para lograr un desarrollo social que no se traduzca en un conflicto de identidad.

El éxito de las políticas multiculturales para lograr su objetivo declarado, que es abatir la inequidad en el acceso a los índices de bienestar, depende, Según Cardoso (2007), del papel que éstas asignen a la diferencia cultural como productora de condiciones de desigualdad social. El autor argumenta el hecho de que, aun cuando queda claro que los organismos internacionales de desarrollo mencionan la diversidad cultural como un factor de desigualdad, refiriéndose a los sistemas de relaciones sociales en los que la diferencia cultural se utiliza para clasificar jerárquicamente a los grupos, es decir, a las relaciones interétnicas, con frecuencia existe una contradicción a la hora de poner en práctica las políticas públicas multiculturales, ya que el interés se pone principalmente en la cultura y no en las relaciones sociales. El problema en México ha sido que, como resultado de ese enfoque, las acciones de gobierno, durante décadas -o sexenios- se centraron, en el mejor de los casos, en difundir el valor de las culturas que se perciben discriminadas, como si la discriminación fuera producto del desconocimiento entre los diversos grupos y no del sistema social.

6 Intentar explicar la desigualdad en razón de la diferencia cultural por sí misma, sin considerarla dentro del sistema de relaciones sociales que la jerarquiza, tiene algunas consecuencias indeseables. Cardoso (2007) enfatiza que esta postura avala políticas de deferencia con indiferencia, porque recicla las teorías que imputan a los desposeídos la causa de su desposesión al obstinarse en perpetuar una cultura inadecuada a la vida moderna, con la notable diferencia de que ahora se les tolera celebrando el derecho a la diferencia. En este sentido, es claro que al resaltar la singularidad de cada grupo cultural, se fomenta la fragmentación extrema de aquellos a quienes la sociedad dominante ha etiquetado como indígenas. El autor enfatiza que, por el contrario, si las políticas multiculturales en verdad pretenden eliminar las asimetrías sociales que se justifican por la diversidad cultural, resulta indispensable entender que la etnicidad es una forma de 
clasificación de las relaciones sociales entre grupos que participan en un mismo sistema social (Cardoso, 2007: 18).

7 Los pueblos indígenas en México han demostrado a través de sus demandas y manifestaciones públicas a lo largo de la historia, la clara conciencia de su derecho a ser contemplados como parte de la estructura social, política y económica del país ${ }^{4}$. Sin embargo, son los gobiernos mexicanos quienes parecen no haber tenido la misma claridad, o teniéndola, no han sido capaces de instrumentar los mecanismos necesarios para lograr la verdadera inserción de esta población en la vida nacional, como sujetos de derecho, en igualdad de circunstancias que el resto de los mexicanos. Aunque aún cabe otra posibilidad: que hayan optado por perpetuar la condición de desigualdad para garantizar el sometimiento de un sector de la población crítico y contestatario, como históricamente lo han sido la mayoría de los pueblos indígenas en México.

\section{El perfil de la diversidad étnica en México}

En México existen 68 etnias, constituidas en 68 pueblos indígenas, a los que corresponden las 68 lenguas que se hablan en el país hasta el día de hoy ${ }^{5}$. Dispersos en diversas comunidades, estos pueblos mantienen sus diferencias culturales, lingüísticas, políticas y religiosas, pero comparten una característica en común que trasciende la multiculturalidad: su incansable y permanente lucha por reclamar la legitimidad de sus derechos. Porque en México, como quizá en muchos otros países, la discriminación ha sido una forma más de asumir el multiculturalismo.

Los grupos indígenas conforman un importante sector de la población mexicana y son claramente identificados como los pueblos originarios del país. Su riqueza histórica y cultural le da sentido de pertenencia e identidad al pueblo de México, y son el tema recurrente de discursos políticos cuando se trata de convocar los valores que promueven el arraigo y la cohesión nacional, o bien cuando se trata de ofertar un producto de consumo para el turismo internacional. Sin embargo, la exaltación de su riqueza, aún por el propio gobierno, no parece coincidir con la posición que éste les asigna en el ámbito de las políticas de desarrollo social, a pesar de ser casi un diez por ciento de la población total del país.

10 En el siguiente cuadro puede observarse un resumen de la densidad de la población indígena y su distribución étnica, lo que implica además, la diversidad de lenguas que se hablan hoy en México.

Cuadro 1

PUEbLOS INDÍgENAS Y LENgUAS PREDOMINANTES EN MÉXICO EN 2010 


\begin{tabular}{|l|r|r|}
\hline & Personas & Porcentaje \\
\hline Población Total & 112336538 & 100 \\
\hline Población Indígena & 11132562 & 9,9 \\
\hline Náhuatl & 2587452 & 23,2 \\
\hline Maya & 1500441 & 13,5 \\
\hline Zapoteco & 771577 & 6,9 \\
\hline Mixteco & 771455 & 6,9 \\
\hline Otomí & 623121 & 5,6 \\
\hline Tseltal & 583111 & 5,2 \\
\hline Tsotsil & 535117 & 4,8 \\
\hline Totonaco & 407617 & 3,7 \\
\hline Mazahua & 336546 & 3,0 \\
\hline Mazateco & 336158 & 3,0 \\
\hline Ch’ol & 283797 & 2,5 \\
\hline Huasteco & 237876 & 2,1 \\
\hline Chinanteco & 207643 & 1,9 \\
\hline Mixe & 194845 & 1,8 \\
\hline Tarasco/Purépecha & 193426 & 1,7 \\
\hline Otrasaneco Lenguas & 1695351 & 12,5 \\
\hline & & \\
\hline & & \\
\hline
\end{tabular}

Fuente: Instituto Nacional de Estadística, Geografía e Informática (INEGI)

11 La población indígena se localiza principalmente en la parte centro y sur del país. En los estados que concentran la mayor cantidad de pobladores indígenas, la proporción rebasa, en promedio, el $20 \%$ de la población total. En el estado de Yucatán rebasa el $50 \%$. La densidad, a nivel de los municipios, es mayor.

De los 624 municipios donde más del 40\% de la población es indígena, en 426 los porcentajes de población en situación de pobreza son superiores al 80\%. Asimismo, 271 municipios indígenas presentan condiciones de pobreza extrema en más del $50 \%$ de su población, concentrados en los estados de Chiapas, Guerrero, Oaxaca y Veracruz ${ }^{6}$.

En todos los casos, las comunidades indígenas se encuentran asentadas en las zonas marginadas del desarrollo urbano ${ }^{7}$. Muchos de ellos incluso lejos de las manchas urbanas, en áreas de difícil acceso, por supuesto sin ningún tipo de servicio básico. Una de las opciones a su alcance ha sido migrar a las ciudades, donde, si les va bien, pueden sumarse a engrosar los cinturones de miseria que rodean a las grandes concentraciones urbanas.

Más allá del discurso de la diversidad y el multiculturalismo como la panacea para implantar -o restaurar- los valores universales del bien común y el respeto a los derechos humanos desde una perspectiva ideológica, que sin duda es importante, se hace necesario articular ese discurso con las acciones concretas del Estado para resolver el drama que subyace, en términos de miseria, desde un proyecto que contemple soluciones estructurales más allá de las prácticas asistenciales que han caracterizado a todos los planes de desarrollo nacional en México.

La diversidad y la multiculturalidad en México, son sinónimos de fragmentación, de debilidad política que cancela cualquier alternativa de desarrollo social auténtico y reproduce los viejos esquemas de la política nacional, con algunos matices en el discurso, que no trascienden a los hechos. 


\section{El perfil institucional mexicano} multiculturalista centrado en el reconocimiento de la diversidad cultural como base de la identidad nacional. Sin embargo, ese reconocimiento se produce en los términos que algunos analistas como Charles R. Hale (Valladares, 2008) han señalado, al afirmar que en el discurso multiculturalista no todas las organizaciones indígenas tienen cabida, en tanto que el reconocimiento de las mismas responde, en gran medida, a los intereses de agencias supranacionales, como el Banco Mundial, para quienes existen dos clases de la expresión de la etnicidad: una, que construye capital social y es digna de ser estimulada, y otra que es disfuncional al modelo neoliberal, porque genera conflictos y disenso. Hale usa el término de "el indio permitido", afirmando que los gobiernos utilizan los derechos propios de la multiculturalidad para dividir y domesticar a los movimientos indígenas. En México, el discurso del multiculturalismo se ha operacionalizado con base en la creación de organismos, comisiones y/o dependencias para atender los problemas de los pueblos indígenas, a partir de un criterio de reconocimiento de su naturaleza identitaria acotado en función de su compatibilidad con el modelo neoliberal.

La crisis provocada por el movimiento zapatista en 1994, por su visibilidad en el plano internacional a través de los medios de comunicación, el activismo del Ejército Zapatista de Liberación Nacional (EZLN), las movilizaciones de apoyo desde diversas partes del mundo, y la presencia de observadores extranjeros en México durante el conflicto y en los años posteriores, motivó la acción del Estado, cuyas propuestas de solución se formularon casi exclusivamente en el aspecto legislativo. Sólo en la década de 1990 y primera mitad de la década de 2000 se realizaron 222 modificaciones, incluyendo constituciones estatales, códigos penales, civiles y sobre justicia indígena, además de diversas leyes en materia de salud, educación, cultura, procuración de justicia, derechos humanos y medio ambiente ${ }^{8}$. Sin embargo, a pesar del trabajo legislativo, las condiciones de desigualdad y exclusión de los pueblos indígenas prevalecen, y en muchos rubros, como el de la pobreza, se ha exacerbado'.

actual gobierno de México ha reconocido, en el discurso oficial, la problemática que enfrentan los pueblos indígenas. El diagnóstico presentado por la Comisión Nacional para el Desarrollo de los Pueblos Indígenas, designada por el actual gobierno, expone en forma detallada las condiciones de marginación desde el ámbito de los derechos humanos, el desarrollo social y económico, así como la condición de exclusión de los pueblos indígenas en las actividades de diseño y coordinación intergubernamental. La propuesta que integra el Programa Especial para los Pueblos Indígenas 2014-2018, parece integrar -en el discurso- las acciones con las que el gobierno del presidente Peña Nieto se propone dar solución a la problemática. Sin embargo, justo en el momento de la redacción de este texto, el 31 de octubre de 2014, aparece en diversos medios la información acerca del Proyecto de Egresos de la Federación, para el 2015, que el presidente Peña Nieto presentó ante la Cámara de Diputados, en la que contempla un incremento al presupuesto destinado para la atención a los pueblos indígenas del $0,47 \%^{10}$. Estos son, entre otras cosas, los hechos. 


\section{Marco metodológico}

19 El análisis de contenido es el conjunto de procedimientos interpretativos, para el estudio de productos textuales de procesos de comunicación previamente registrados, que basados en técnicas de medición -cuantitativas y/o cualitativas- según Piñuel (2002), tienen por objeto elaborar y procesar datos relevantes sobre las condiciones en que se han producido aquellos textos, o sobre las condiciones que puedan darse para su empleo posterior. De acuerdo con el autor "los textos remiten a un universo de prácticas sociales y cognitivas, donde cualquier interacción comunicativa cobra unos límites particulares que son mediados y mediadores de aquellas prácticas a las que sirve".

El presente análisis, se enfoca en el Programa Especial de los Pueblos Indígenas 2014-2018 ${ }^{11}$, como producto textual de un largo proceso de comunicación (que se describe en el apartado teórico de este trabajo), cuyos interlocutores reconoceremos en forma genérica como: la población indígena y el Estado Mexicano, con el objeto de procesar algunos datos relevantes acerca de las condiciones en que éste se produjo.

Como corresponde a un análisis de contenido, el objetivo es lograr la emergencia de ese sentido latente que trasciende la superficie material del texto y que procede de las prácticas sociales e institucionales, que recurren al discurso oficial -legal- para facilitar su interacción.

22 Como señala Bardin (en Piñuel y Gaitán, 1995), el análisis de contenido se convierte en una empresa de des-ocultación o revelación de la expresión, donde, ante todo, interesa indagar sobre lo escondido, lo latente, lo no aparente, lo potencial, lo inédito (lo no dicho) de todo mensaje. Desde esta perspectiva, la intención fue ampliar el campo de estudio, hacia una dimensión no manifiesta del Programa Especial de los Pueblos Indígenas 2014-2018 que, por ser una construcción compleja, requirió de la definición de variables que orientaran la búsqueda desde las condiciones contextuales y, por lo tanto, desde las circunstancias de producción y recepción de dicho documento. En ese sentido, se abordó el análisis a partir del modelo de la Mediación Dialéctica de la Comunicación Social (MDCS) que, de acuerdo con Piñuel (2002), permite formular un sistema conceptual especializado en la descripción, explicación y predicción de los cambios que experimentan o pueden experimentar los sistemas de comunicación propios de nuestras sociedades.

Este modelo incorpora los avances teóricos de diversos campos, entre los que destacan: la Dialéctica, y la Teoría de Sistemas, para ampliar la observación y el análisis desde una perspectiva que reconoce la complejidad de los fenómenos y facilita la comprensión de los procesos sociales, sobre todo de aquéllos en los que la Comunicación se encuentra implicada.

24 Para aplicar el MDCS, es necesario partir de la idea de que un sistema de comunicación social, y los constantes cambios que experimenta, no pueden ser percibidos si no se establece primero una clara diferenciación entre los componentes y relaciones que son específicos del propio sistema de comunicación (SC), y los componentes y relaciones que son específicos de otros sistemas. Piñuel, abunda en la explicación de la siguiente manera:

Una vez efectuada esa diferenciación, los cambios de los sistemas d comunicación (SC) sólo pueden explicarse examinando las relaciones de apertura que dicho sistema mantiene con otros dos sistemas generales: el sistema social (SS) y el sistema ecológico-adaptativo (SE), cuyo específico inventario de componentes genéricos y de relaciones internas (estructurales y funcionales) es igualmente 
proporcionado por dicho modelo (cfr. Martín Serrano et al., 1981; Martín Serrano, 1989; Piñuel, 1989 y Piñuel \& Gaitán, 1995). La concepción de los sistemas de comunicación (SC), de los sistemas sociales (SS) y de los sistemas ecológicoadaptativos (SE) como sistemas abiertos entre sí, nos permite describir la transformación de cada uno de esos tres sistemas en términos de una mediación dialéctica intersistémica, es decir, concibiendo la transformación interna de un determinado sistema como el producto de la influencia externa que ejercen sobre él los sistemas restantes, y viceversa. (Piñuel, 2002: 5)

Con base en estadísticas centradas en el recuento de datos (técnicas cuantitativas), y procedimientos lógicos basados en la combinación y contrastación de categorías (técnicas cualitativas) fue posible elaborar y procesar algunos datos relevantes sobre las condiciones en que el Programa Especial de los Pueblos Indígenas 2014-2018 fue producido, y las que pudieran presentarse en su posterior ejecución.

gorías de análisis en las que se basó el procedimiento interpretativo, fueron diseñadas como una adaptación del modelo de la MDCS, al objetivo de este trabajo, cuyos componentes se asumen de la siguiente manera:

1. El Sistema de Comunicación. Entendido como el Programa Especial de los Pueblos Indígenas 2014-2018, en su calidad de producto textual de un proceso de comunicación, que se convierte a su vez en un sistema que facilita la interacción mediada del Estado mexicano y la población indígena en el país.

2. El Sistema Social. Entendido como el conjunto de instituciones, organismos y procesos de la sociedad civil, que determinan la superestructura y los patrones de comportamiento de la población en general.

3. El Sistema Ecológico-Adaptativo. Entendido como el conjunto de instituciones, organismos y procesos de la sociedad civil, que operan en favor de una sostenibilidad, de largo plazo, en la relación del sistema de comunicación y el sistema social.

Más allá de la identificación intra-textual de la estructura que adopta el Programa Especial de los Pueblos Indígenas 2014-2018, lo más interesante resulta ser el conocimiento que se produce a partir de los contextos donde se inscribe este material de análisis, así como la descripción de las situaciones y entornos implicados en su producción, y la identificación de las estructuras que rigen la conducta de los individuos, grupos, o instituciones emisoras y receptoras, en los procesos de comunicación que le dieron origen. Se trata pues, de un análisis de contenido vertical (de un solo caso), descriptivo, a un objeto de estudio refutable, y desarrollado a partir de fuentes documentales primarias.

\section{Resultados del análisis}

\section{Modelo de Mediación Dialéctica de la Comunicación}

\section{CUADRO 1}

SISTEMA DE COMUNICACIÓN (SC)

PROgRAMA ESPECIAL DE LOS PUEblos Indígenas 2014-2018 


\begin{tabular}{|c|c|c|}
\hline \multirow{2}{*}{$\begin{array}{l}\text { Componentes } \\
\text { genéricos }\end{array}$} & \multicolumn{2}{|c|}{ Relaciones internas } \\
\hline & Estructurales & Funcionales \\
\hline $\begin{array}{l}\text { Mensaje: } \\
\text { Plan de trabajo de } \\
\text { nivel Federal }\end{array}$ & $\begin{array}{l}\text { Tiene un carácter oficial, } \\
\text { al ser aprobado por } \\
\text { decreto presidencial. }\end{array}$ & \multirow{2}{*}{$\begin{array}{l}\text { Se opera a través de la Comisión } \\
\text { Nacional para el Desarrollo de } \\
\text { los Pueblos Indígenas, como } \\
\text { responsable del seguimiento y } \\
\text { ejecución de las acciones. }\end{array}$} \\
\hline $\begin{array}{l}\text { Actores: } \\
\text { Estado Mexicano } \\
\text { Población indígena }\end{array}$ & $\begin{array}{l}\text { Es de observancia } \\
\text { obligatoria para todos } \\
\text { los niveles de gobierno. }\end{array}$ & \\
\hline $\begin{array}{l}\text { Lenguaje: } \\
\text { Discurso Oficial }\end{array}$ & \multirow{2}{*}{$\begin{array}{l}\text { Posee autoridad, con } \\
\text { injerencia en todas las } \\
\text { dependencias de la } \\
\text { administración pública } \\
\text { relacionadas con la } \\
\text { población indígena. }\end{array}$} & \multirow{2}{*}{$\begin{array}{l}\text { Comparte responsabilidad con } \\
\text { la Secretaría de Hacienda y } \\
\text { Crédito Público, y la Secretaría } \\
\text { de la Función Pública, para el } \\
\text { cumplimiento de los objetivos y } \\
\text { la rendición de cuentas. }\end{array}$} \\
\hline $\begin{array}{l}\text { Medio: } \\
\text { Política Pública }\end{array}$ & & \\
\hline
\end{tabular}

Cuadro 2

Sistema Social (SS) 


\begin{tabular}{|c|c|c|}
\hline \multirow{2}{*}{$\begin{array}{c}\text { Componentes } \\
\text { genéricos }\end{array}$} & \multicolumn{2}{|c|}{ Relaciones internas } \\
\hline & Estructurales & Funcionales \\
\hline $\begin{array}{l}\text { La estructura oficial: } \\
\text { Antecedentes } \\
\text { históricos de la } \\
\text { política nacional, } \\
\text { en relación con la } \\
\text { atención a los pueblos } \\
\text { indígenas, en los } \\
\text { últimos treinta años. } \\
\text { 1. En el discurso } \\
\text { 2. En los hechos } \\
\text { La respuesta colectiva } \\
\text { de los pueblos } \\
\text { indígenas: } \\
\text { Antecedentes } \\
\text { históricos del } \\
\text { movimiento indígena, } \\
\text { en los últimos treinta } \\
\text { años. } \\
\text { 1. En el discurso } \\
\text { 2. En los hechos }\end{array}$ & $\begin{array}{l}\text { Centralización de los } \\
\text { programas federales, como } \\
\text { condición que retrasa o } \\
\text { inhibe la cobertura y la } \\
\text { aplicación de los recursos. } \\
\text { Autonomía de los poderes } \\
\text { Estatal y Municipal, que } \\
\text { en ocasiones no favorece la } \\
\text { coordinación y la suma de } \\
\text { esfuerzos. } \\
\text { Escasa o nula relación } \\
\text { formal entre los niveles de } \\
\text { gobierno. } \\
\text { Escaso presupuesto. } \\
\text { Falta de continuidad de los } \\
\text { programas y políticas públi- } \\
\text { cas, de un sexenio a otro. } \\
\text { Falta de mecanismos } \\
\text { formales de evaluación y } \\
\text { seguimiento. }\end{array}$ & $\begin{array}{l}\text { Ideologías e intereses } \\
\text { partidistas diversos y, en } \\
\text { ocasiones, antagónicos*. } \\
\text { Con frecuencia se ponderan } \\
\text { acuerdos y alianzas por } \\
\text { encima de las disposiciones } \\
\text { constitucionales**. } \\
\text { Burocracia } \\
\text { Corporativismo } \\
\text { Baja calidad en la } \\
\text { educación*** } \\
\text { Existe un sesgo en la } \\
\text { educación relacionado } \\
\text { con la posición } \\
\text { económica*** }\end{array}$ \\
\hline
\end{tabular}

* EXISTEN INNUMERABLES REgISTROS EN NOTAS DE PRENSA Y MEDIOS ELECTRÓNICOS, QUE DAN CUENTA DE CONTROVERSIAS ENTRE LOS REPRESENTANTES DE LOS DIVERSOS PARTIDOS. ALgUNAS DE ELLAS HAN LLEgADO A TRASCENDER AL INTERIOR DEL CONgRESO DIfICULTANDO LOS PROCESOS DE DIÁLOgO.

** POCO DESPUÉs de SU TOMA DE PROTESTA COMO PRESIDENTE dE MÉXICO, ENRIQUE PEÑA NIETO PROPUSO LA CREACIÓN DE UN gRUPO ALIADO, CON REPRESENTACIÓN DE LOS PARTIDOS MAYORITARIOS, TOMANDO DECISIONES QUE SON COMPETENCIA DEL CONgRESO, AL MARgEN DE ÉSTE. *** PUEDEN CONSULTARSE LOS INDICADORES OfICIALES EN: HTTP://WWW.ENLACE.SEP.gOB.MX/ CONTENT/gR/DOCS/2013/HISTORICO/00_EB_2013.PDF.

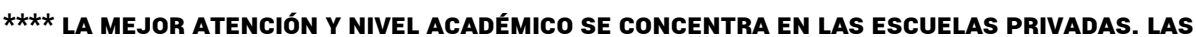
ESCUELAS PÚBLICAS NO TIENEN LA CAPACIDAd SUfiCIENTE PARA ATENDER LA DEMANDA Y EL NIVEL ACADÉMICO, POR LO gENERAL, ES BAJO.

Cuadro 3

Sistema Ecológico Adaptativo (SEA) 


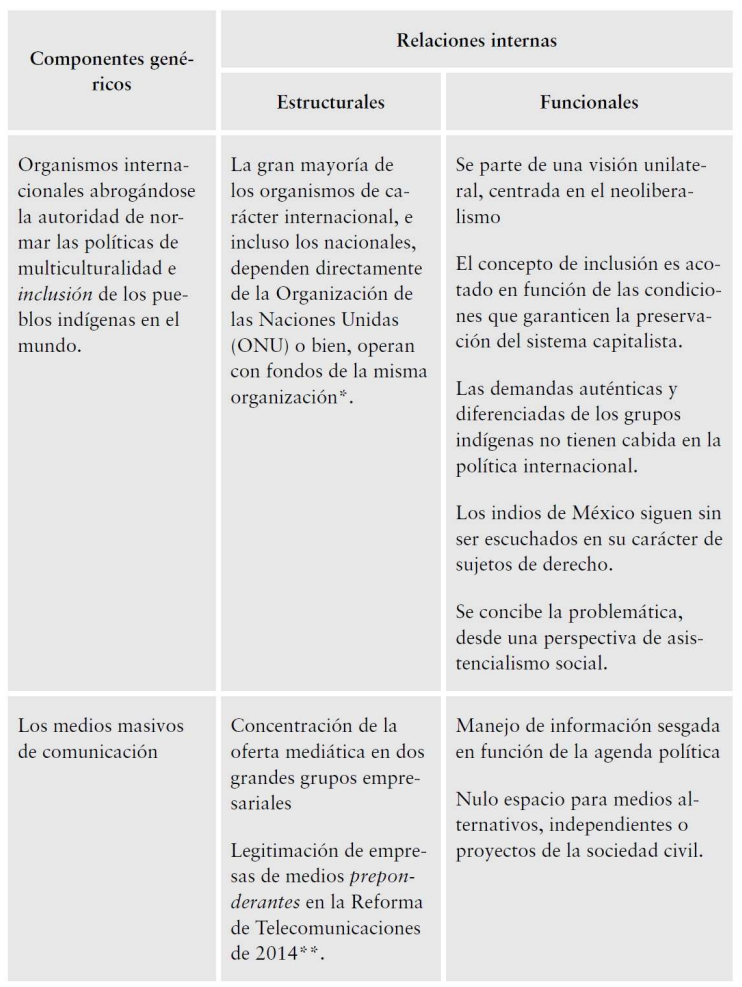

* PUEDE REVISARSE EL INFORME SOBRE LAS ACCIONES DESARROLLADAS POR DIFERENTES ORgANISMOS INTERNACIONALES PARA LA PROMOCIÓN DE LOS DERECHOS DE LOS PUEBLOS INDÍgENAS, HASTA EL AÑO DE 1999 EN: HTTP://WWW.SUMMIT-AMERICAS.ORg/INDIGENOUS/W-GROUPOCT99/INSTITUTO\% 20INDIgENISTA\%20AMERICANO.HTM.

** Ver el Diario Ofi cial de la Federación del 14 de julio de 2014, disponible en: http://www.dof.gob.mx/ nota_detalle.php?codigo=5352323\&fecha=14/07/2014

\section{El sistema social en la relación Estado-población indígena}

31 Entender a cabalidad la naturaleza de la relación del Estado y la población indígena en México, demanda un análisis a profundidad del conjunto de relaciones, naturales e impuestas, que ambos han construido a lo largo de la historia; esa perspectiva ha sido abordada puntualmente por diversos autores, como: Valladares (2008), Bárcenas (2004), Gómez (2004), Knight (1995), Sarmiento y Mejía (1988) y Reyna (1980), entre otros.

En la actualidad, esa relación se produce en medio de las tensiones que el sistema-mundo introduce en el terreno de la economía, los derechos humanos, la política de multiculturalidad -que, más que responder a las necesidades de los pueblos indígenas, parece orientada a normar criterios-, además de una serie de contingencias políticas que elevan las dificultades para lograr el óptimo desarrollo de los pueblos, en términos de bienestar social, especialmente cuando se vive en una situación de economía precaria, como es el caso de México. En este proceso, los esfuerzos por entablar una comunicación abierta, fluida y productiva, parecen ser, en primera instancia, el recurso más valioso para lograr la armonización de intereses y el establecimiento de acuerdos, que si bien no son suficientes para resolver los problemas de fondo, sí lo son para crear las condiciones propicias que faciliten la búsqueda de soluciones concretas. Sin embargo,

El proceso de reforma del Estado que ha vivido el país, ha significado el fin del

Estado de bienestar social. El mexicano fue un Estado cuya política social, heredada de

la revolución de 1910, lo situó como paternalista, subsidiador (sic) del desarrollo y 
redistribuidor de la riqueza. Sin embargo, a partir de la reforma del Estado y con el argumento de su ineficiencia por sus prácticas corruptas y clientelares en la distribución de los recursos federales, así como por su incapacidad para pagar la deuda externa, su ineficiencia en la administración de las más de 1.000 empresas que poseía, se dio inicio al desmantelamiento de una estructura económica y política incapaz de generar un crecimiento, tanto a nivel macro económico, como para revertir el proceso de empobrecimiento de amplias capas sociales. (Valladares, 2008: 291) comprender la naturaleza intrínseca de la actual relación: Estadopoblación indígena. Es decir, no sería posible entender el proceso de comunicación que el gobierno de Enrique Peña Nieto pretende entablar con los pueblos indígenas a través de un aparato burocrático político-administrativo, cuya operación depende en última instancia de la Secretaría de Gobernación ${ }^{12}$, si no tenemos una mínima referencia del pasado, por lo menos del pasado reciente, en la historia de esa relación. En este sentido, resulta particularmente interesante la perspectiva de Castellanos (2000: 7), cuando dice:

Las relaciones entre etnia, Estado y nación tienen su origen en múltiples experiencias históricas, particularmente en el colonialismo y en distintos momentos del proceso de formación de las naciones modernas y de la expansión capitalista en sus diversas modalidades; por ello, examinar el contexto histórico es condición para comprender su naturaleza.

A continuación se presenta una breve síntesis de los puntos de encuentro y desencuentro en la relación del Estado mexicano y sus pueblos indígenas, durante los últimos treinta años, que han impregnado la vida nacional de un clima de tensión que aún sigue vigente. Los datos se presentan a partir de dos variables: 1) La política social en materia de derechos indígenas de los cinco períodos de gobierno que antecedieron a la administración de Enrique Peña Nieto y 2) El movimiento indígena, durante el mismo período.

La información en cada una de las variables se presenta desde dos indicadores: el discurso, cuyos datos fueron extraídos de documentos oficiales, en su calidad de fuentes primarias, y que consisten, por un lado: en los Planes de Desarrollo que cada presidente hizo público al inicio de su mandato, y por el otro: los comunicados provenientes de la dirigencia del movimiento indígena ${ }^{13}$. segundo indicador en cada variable se denomina los hechos, cuya información fue extraída de registros hemerográfi cos y documentos públicos del Centro de Estudios Latinoamericanos ${ }^{14}$. Se trata de esquematizar la relación sincrónica y diacrónica de los discursos y los hechos, de los dos actores sociales que protagonizan, en México, la historia imperfecta de la multiculturalidad.

Cuadro 4

LA RELACIÓN ESTADO-POBLACIÓN INDígENA, EN MÉXICO, DE 1982 A 1988 


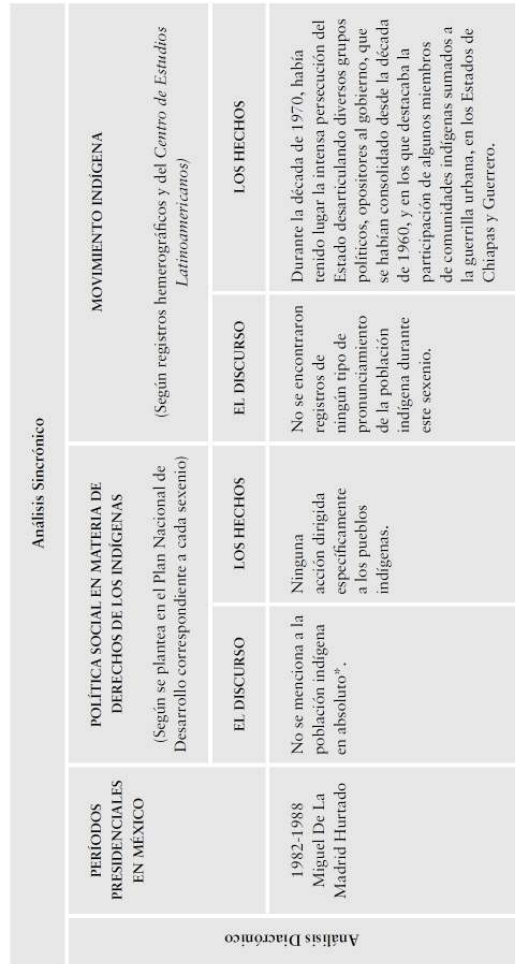

* EL ENFOQUE PRIORITARIO EN TOdOS LOS RUBROS ESTÁ CENTRADO EN MEDIDAS ECONÓMICAS. SU PRIORIDAD ES ABATIR LA CRISIS Y RELACIONARSE CON MERCADOS INTERNACIONALES.

Cuadro 5

LA RELACIÓN ESTAdO-POBLACIÓN INDÍgENA, EN MÉXICO, DE 1989 A 1994

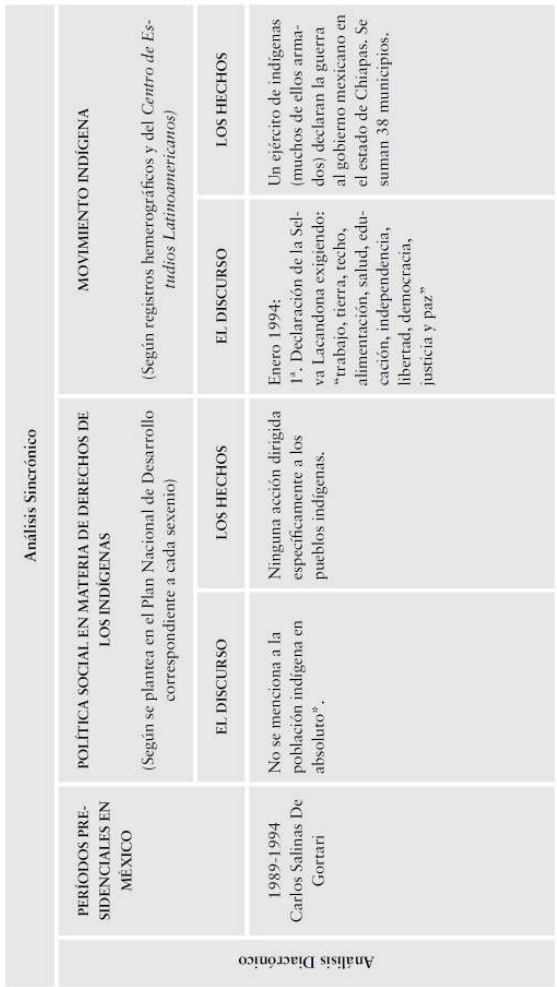




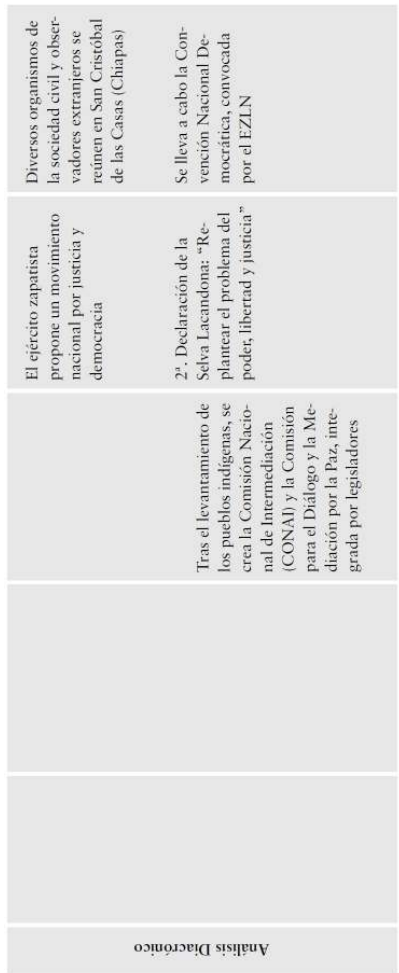

* Lo más cercano es un plan de modernización en el medio RURAL a fin de contener La MIgRACIÓN RURAL A CENTROS URBANOS.

Cuadro 6

LA RELACIÓN ESTADO-POBLACIÓN INDÍgENA, EN MÉXICO, DE 1995 A 2000

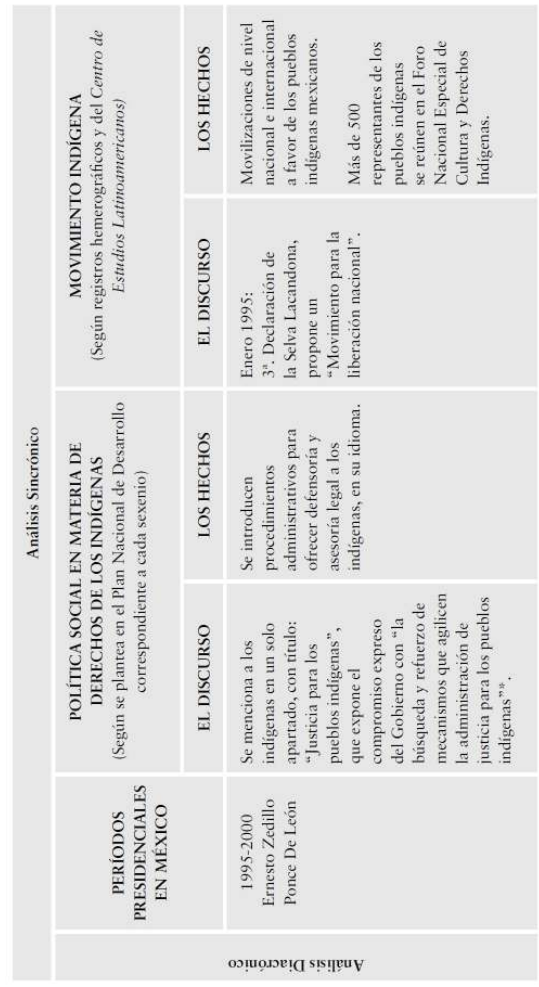



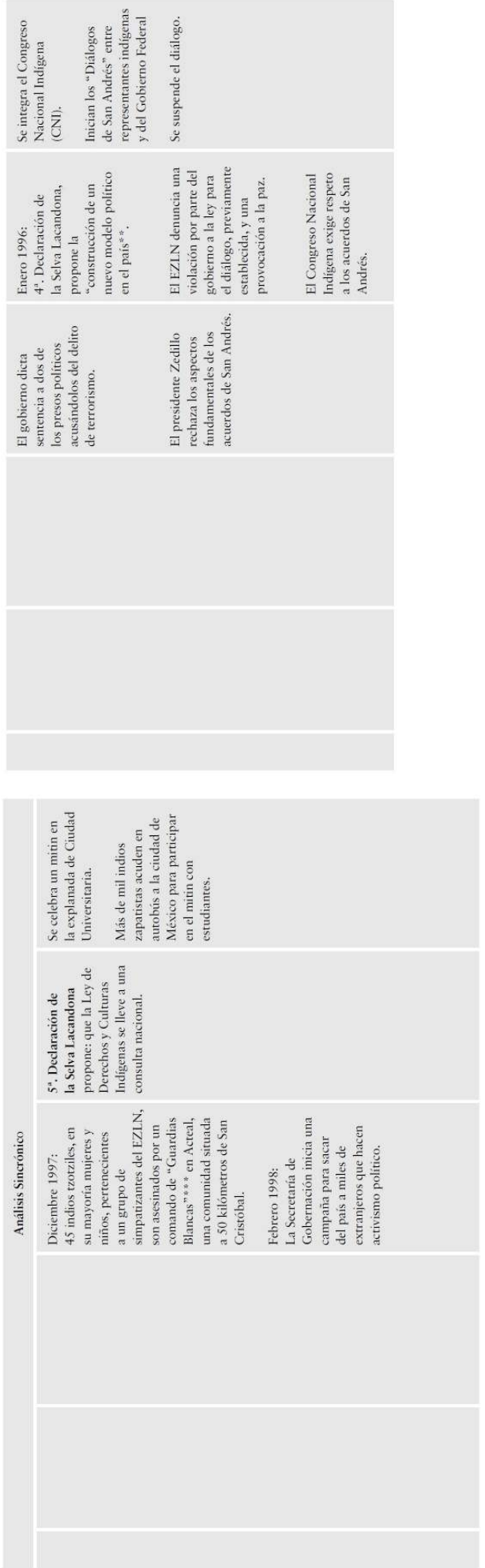


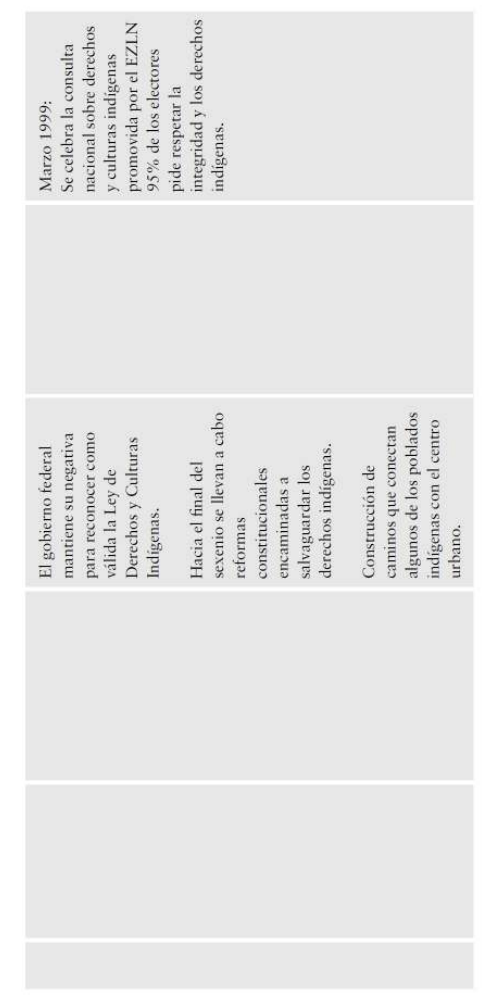

* Los MENCIONAdOS ESFUERzos SE desglosan ALUdiendo A LA FORMACIÓN DE INTÉRPRETES EN LENGUAS INDÍgENAS, Y LA GARANTÍA DE CONTAR CON UN TRADUCTOR EN TODOS SUS PROCESOS LEgALES, ASÍ COMO “REALIZAR UNA MAYOR Y MÁS EFICAZ DIFUSIÓN DE SUS DEBERES Y DERECHOS Y DE LOS PROCESOS JURÍDICOS QUE LES ATAÑEN".

** ARgUMENTA LA VIABILIDAD DE UNA ESTRUCTURA NO PARTIDARIA, INDEPENDIENTE, AUTÓNOMA Y PAcíficA.

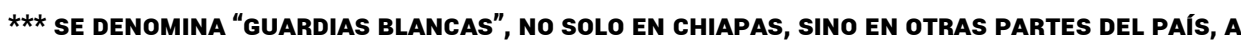
LOS gRUPOS ARMADOS AL SERVICIO DE gRANDES LATIFUNDISTAS, CUYA FUNCIÓN ES PROTEgER A ÉSTOS dE INVASIONES POR PARTE DE EJIDATARIOS O INDÍgENAS. EN EL CASO CONCRETO DE CHIAPAS, LAS "GUARDIAS BLANCAS" ESTÁN AL SERVICIO DE LOS DUEÑOS DE HACIENDAS CAFETALERAS QUE POSEEN MILES DE HECTÁREAS DE LAS MEJORES TIERRAS EN ESE ESTADO. LA MAYORÍA DE LOS PROPIETARIOS DE LAS HACIENDAS CAFETALERAS SON EXTRANJEROS Y ALgUNOS MEXICANOS DESCENDIENTES DE EXTRANJEROS, QUE VIVEN EN PAíses EUROPEOS.

Cuadro 7

LA RELACIÓN ESTADO-POBLACIÓN INDígENA, EN MÉXICO, DE 2001 A 2006 


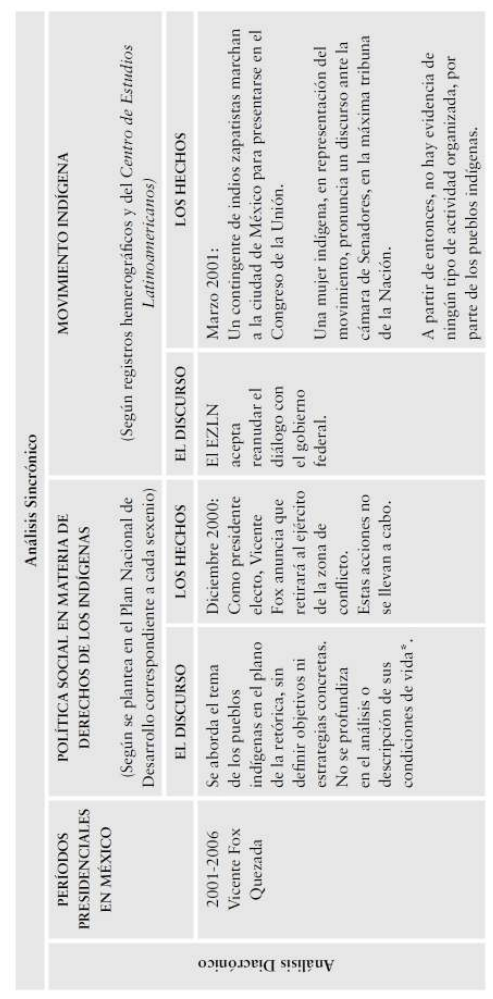

“APOYAR EL PROCESO DE DESARROLlO INTEgRAL INDÍgENA DENTRO DEL CONTEXTO NACIONAL CREANDO LAS CONDICIONES PARA LOgRAR UNA COMUNICACIÓN E INTERACCIÓN EFECTIVA CON DIVERSOS SECTORES DE LA SOCIEDAD, ADEMÁS DE ESTABLECER PROgRAMAS gUBERNAMENTALES DE gRAN TRASCENDENCIA QUE COMBATAN LOS PROBLEMAS ESTRUCTURALES QUE HAN OBSTACULIZADO $O$ LIMITADO EL DESARROLLO DE LOS PUEBLOS INDÍgENAS, CON EL PROPÓSITO DE MEJORAR SU NIVEL DE VIDA"...."PROPICIAR LA PARTICIPACIÓN DIRECTA DE LOS PUEBLOS INDÍgENAS EN EL DESARROLLO NACIONAL Y COMBATIR LOS REZAgOS Y LAS CAUSAS ESTRUCTURALES DE SU MARgINACIÓN CON PLENO RESPETO A SUS USOS Y COSTUMBRES".

Cuadro 8

LA RELACIÓn ESTAdO-POBLACIón INDígENA, EN MÉXICO, DE 2007 A 2012 


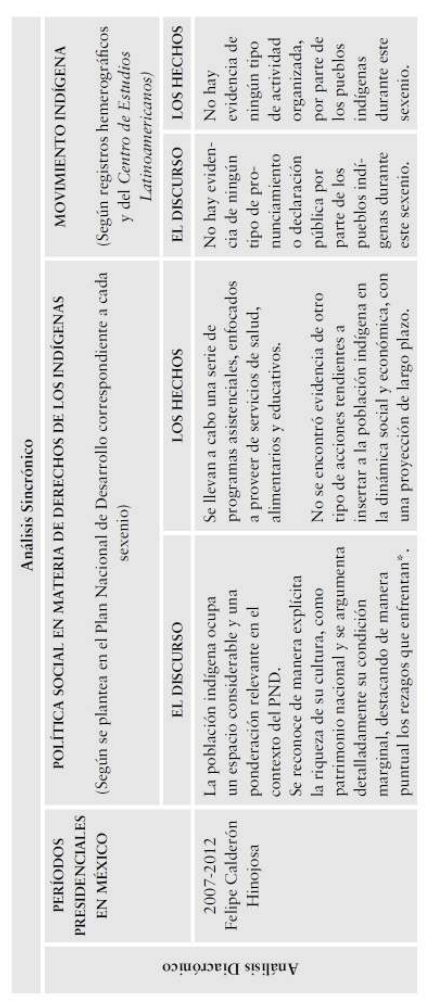

* SE SEÑALA PARTICULARMENTE: LA DESNUTRICIÓN Y DEfICIENCIAS NUTRICIONALES, LA MORTALIDAD Y MORBILIDAD, EL REZAgO EDUCATIVO, LA DESIgUALDAD DE gÉNERO, Y LOS PROBLEMAS DERIVADOS DE LA MIgRACIÓN. "DESgRACIADAMENTE, LOS PUEBLOS Y COMUNIDADES INDÍgENAS AÚN NO DISFRUTAN DE UNA SITUACIÓN SOCIAL Y ECONÓMICA PROPICIA PARA EL MEJOR DESARROLLO HUMANO; SE CARACTERIZAN POR VIVIR EN ALTOS NIVELES DE POBREZA Y EN UNA SITUACIÓN DE SIgNIfiCATIVA DESVENTAJA. EL COMBATE AL REZAgO SOCIAL DE LOS PUEBLOS Y LAS COMUNIDADES INDÍgENAS REPRESENTA UNA DE LAS ÁREAS DE POLÍtiCA PÚbliCA DE MAYOR RELEVANCIA PARA EL DESARROLLO ARMÓNICO DE MÉXICO. ES FUNDAMENTAL QUE LAS POLÍTICAS PÚBLICAS QUE IMPULSE EL GOBIERNO FEDERAL, EN COORDINACIÓN CON LAS ENTIDADES FEDERATIVAS Y LOS MUNICIPIOS, TENGAN UN CARÁCTER ESPECÍ́fiCO EN LA PERSPECTIVA INTEgRAL DEL DESARROLLO HUMANO SUSTENTABLE PARA INCREMENTAR LAS CAPACIDADES DE LOS PUEBLOS Y LAS COMUNIDADES INDÍgENAS". SE PLANTEA COMO OBJETIVO: "INCORPORAR PLENAMENTE A LOS PUEBLOS Y A LAS COMUNIDADES INDÍgENAS AL DESARROLLO ECONÓMICO, SOCIAL Y CULTURAL DEL PAíS CON RESPETO A SUS TRADICIONES HISTÓRICAS Y ENRIQUECIENDO CON SU PATRIMONIO CULTURAL A TODA LA SOCIEDAD.

LA PRIMERA DE SIETE ESTRATEgIAS ES: "CONSTITUIR LA ATENCIÓN A LOS INDÍgENAS EN UN OBJETIVO ESTRATÉgICO TRANSVERSAL PARA TODA LA ADMINISTRACIÓN PÚBLICA FEDERAL"

\section{El Programa Especial de los Pueblos Indígenas (PEPI) 2014-2018}

\subsection{El discurso}

La descripción de la Nueva política de inclusión y desarrollo de los Pueblos Indígenas en los propios términos del PEPI plantea lo siguiente:

La visión que el Gobierno Federal tiene para el horizonte sexenal es que los pueblos y comunidades indígenas vivan en condiciones sociales y económicas dignas, dentro de un marco de igualdad e interculturalidad.

El Gobierno de la República impulsa políticas públicas que respetan sus derechos y su identidad, que propician la participación y la autogestión de sus procesos de desarrollo. La política hacia el sector se caracteriza por promover la transversalidad y la integralidad de las propuestas y acciones de política pública.

Particularmente, en el marco del Plan Nacional de Desarrollo 2013-2018, la Meta Nacional 2, "México Incluyente", engloba los objetivos y las acciones dirigidas a 
combatir la pobreza y la desigualdad. El objetivo es: hacer realidad un país donde se asegure el ejercicio efectivo de los derechos sociales, económicos y culturales.

El Gobierno de la República se plantea impulsar una sociedad con equidad, cohesión social e igualdad de oportunidades. Esto es una política social de nueva generación, enfocada en alcanzar una sociedad de derechos ciudadanos y humanos plenos que contribuya a cerrar las brechas sociales. Esto implica hacer efectivo el ejercicio de los derechos sociales de todos los mexicanos, a través del acceso a: servicios básicos, agua potable, drenaje y saneamiento, electricidad, seguridad social, educación, alimentación y vivienda digna.

Este objetivo es más relevante cuando se refiere a la población indígena, de ahí que la Comisión Nacional para el Desarrollo de los Pueblos Indígenas tiene la responsabilidad de orientar eficazmente, las políticas, los programas y proyectos, del gobierno hacia la promoción del desarrollo integral y sustentable de los pueblos y comunidades indígenas.

Existe un compromiso del Gobierno Federal para mejorar las condiciones de vida de la población indígena y combatir esta realidad de pobreza y marginación, a través de políticas y acciones públicas que les permitan a los Pueblos Indígenas tener acceso a mejores condiciones de educación, salud, vivienda, infraestructura básica e ingreso.

39 Con este discurso como base, y después de un ejercicio de consulta y validación de su Consejo Consultivo, según lo expuesto en el documento oficial, la CDI definió una estrategia de trabajo considerando cinco ejes estratégicos de atención:

- Derechos Indígenas y Acceso a la Justicia, impulsando la armonización legislativa, el apoyo legal a indígenas encarcelados o sujetos a algún tipo de proceso penal, agrario o administrativo. Desarrollo Social, a través del cual se dotará de más y mejor infraestructura básica a las comunidades y hogares indígenas, además de atender las necesidades de alimentación, salud, educación, vivienda e infraestructura básica.

41 - Desarrollo Económico, el cual considera la promoción de fuentes de ingreso monetario y no monetario para las comunidades y familias indígenas.

42 - Participación de la sociedad indígena y coordinación intergubernamental, en la planeación y gestión del desarrollo de los Pueblos Indígenas.

43 - Preservación y fomento de la cultura de los Pueblos Indígenas como patrimonio nacional y elemento articulador de los otros cuatro ejes de atención.

\subsection{Los hechos}

44 Aunque existen evidencias de la actividad de la Comisión Nacional para el Desarrollo de los Pueblos Indígenas en la cobertura informativa de algunos medios nacionales, sobre todo en lo que se refiere a las reuniones de acuerdos sostenidas entre el representante de dicha comisión y grupos de representación indígena, no se dispone, a la fecha, de información oficial de carácter público, que nos permita elaborar una valoración sobre el avance en el logro de sus objetivos, lo cual es comprensible dado el corto período de tiempo transcurrido desde el inicio de la gestión de este organismo.

Sin embargo, a partir del análisis de contenido desde la Mediación Dialéctica de la Comunicación Social, se identifican algunos elementos contextuales, que de manera natural introducen una idea de escepticismo con relación a la viabilidad del programa, que tienen que ver principalmente con las condiciones económicas, sociales y políticas por las que atraviesa hoy el Estado mexicano y que se abordan a continuación. 


\section{Conclusiones}

46 el compromiso de velar por la democracia y el buen manejo de los recursos federales, ha
sido retomado por todos los presidentes, sin excepción, desde hace poco más de cuatro décadas; mientras las condiciones de pobreza, la marginación y los conflictos internos de la clase política -muchos de ellos evidenciando actos de corrupción- siguen en aumento. El tema de la multiculturalidad que aparece en la agenda política, como uno de los rubros de mayor interés para el gobierno, no acaba de definirse en términos de una estrategia clara para asumir esa realidad, desde una estructura social, política y económica capaz de armonizar las diferencias en términos de igualdad de oportunidades y justicia para todos.

Aunque los derechos de los indígenas son reconocidos en el Programa Especial de los Pueblos Indígenas 2014-2018, no se percibe un esfuerzo paralelo del Estado, ni la voluntad política para generar las condiciones propicias que garanticen su ejecución desde el punto de vista institucional. El desconocimiento en materia legislativa por parte de la población indígena, los procedimientos burocráticos, el escaso dominio del tema por el personal encargado de dichos procesos, la dificultad que impone el idioma y los candados prediseñados para coptar la autonomía de los pueblos indígenas, desalienta las iniciativas de búsqueda de soluciones a través de la vía institucional ${ }^{15}$.

El diagnóstico que presenta la Comisión Nacional para el Desarrollo de los Pueblos Indígenas, da cuenta de una realidad que se retoma medianamente en el planteamiento de los objetivos y el diseño de estrategias del Programa Especial de los Pueblos Indígenas 2014-2018, pues no contempla ningún tipo de acciones tendientes a promover los cambios estructurales necesarios, limitándose a reproducir las prácticas asistencialistas.

La oferta de servicios que el Programa plantea en favor de las comunidades indígenas, el nivel de gestión implicado y la magnitud de la cobertura necesaria, no sólo por la cantidad de comunidades indígenas que existen en México, sino por su dispersión geográfica a lo largo del territorio nacional, demandan una fuerte inversión económica. En este sentido, la propuesta del $0,47 \%$ de incremento presupuestal designado a este rubro presentada por el presidente Peña Nieto al Congreso, sólo puede considerarse como un dato que permite comprobar -en los hechos- la falta de voluntad política y la nula viabilidad del mencionado programa.

El bajo nivel educativo, en todos los niveles de formación, que se registra en los propios parámetros del gobierno federal, nos dan un indicio de la problemática originaria de una larga cadena de impactos visibles en la sociedad mexicana, que inicia con una producción en serie de ciudadanos poco informados y poco ejercitados en la práctica de las habilidades cognitivas que garanticen un perfil crítico de su entorno. Por lo tanto, la búsqueda de programas educativos de mayor calidad por parte de un sector de la población, se orienta a la oferta de las escuelas privadas, creando un sesgo en la educación relacionado con la posición económica. Este solo hecho, contribuye a polarizar la desigualdad social. En estas condiciones, en México, se vuelve extremadamente difícil paliar la pobreza y la desigualdad a través de la educación, y el acceso a ésta, en el caso de los indígenas, sigue siendo una asignatura pendiente.

51 Por otra parte, la permisividad del gobierno mexicano hacia los medios de comunicación, particularmente las televisoras, que hace posible la existencia de monopolios de la 
información y de una oferta mediática que pondera el entretenimiento, han abonado a la formación de individuos con una escasa conciencia social que, a su vez, se traduce en prácticas individualistas y en una escasa -o nula- participación de la ciudadanía en los procesos de transformación que requiere el país.

Ante este panorama se radicalizan, no sólo las posiciones de los grupos inconformes, sino las medidas represivas del Estado, en una lucha de fuerzas a todas luces desigual -en términos de poder-, donde las protestas de grupos conscientes de la sociedad civil, incluidos los grupos indígenas, buscan compensar este desequilibrio recurriendo a cobrar visibilidad en la esfera pública, logrando la solidaridad internacional, y afectando la imagen de los diferentes niveles de gobierno, principalmente en los Estados del sur. Los medios de comunicación con mayor poder de cobertura -los monopolios alineados con el gobierno- contribuyen a la represión ejercida por el Estado mexicano, aportando una versión sesgada de las protestas e induciendo una imagen de vandalismo y delincuencia que el gobierno utiliza para justificar la represión violenta, con el aval de los medios a su favor ${ }^{16}$.

Retomando el sentido del análisis de contenido al Programa Especial para los Pueblos Indígenas 2014-2018, aprobado por decreto presidencial el 30 de abril de 2014, desde el marco contextual que determina la Mediación Dialéctica de la Comunicación Social, puede afirmarse que existe un alto nivel de incongruencia entre la expresión intra-textual y la realidad contextual. Es decir, entre los objetivos que el Estado dice perseguir, y la evidencia de sus acciones ante las demandas de la misma población a la que dice proteger.

El sistema ecológico adaptativo, representado en este análisis -además de los medios de comunicación- por los diversos organismos internacionales que norman las políticas de multiculturalidad e inclusión de los pueblos indígenas en el mundo, no logran establecer las condiciones para una relación sustentable entre el Estado mexicano y su población indígena, poniendo en relieve la necesidad de un diálogo renovado y un esfuerzo de negociación que atienda los aspectos fundamentales del debate, con una vocación conciliadora auténtica, desde una perspectiva humana, más que política.

\section{BIBLIOGRAFÍA}

BOCCARA, Guillaume y BOLADIS, Paola (2010), “¿Qué es el multiculturalismo? La nueva cuestión étnica en el chile neoliberal”, Revista de Indias. Vol. LXX. Num. 250, pp. 651-690. ISSN: 0034-8341 doi:10.3989/revindias.2010.021.

CARDOSO DE OLIVEIRA, Roberto (2007), Etnicidad y Estructura Social. UAM-UIA-CIESAS. México.

CERDA, Alejandro (2007), "Multiculturalidad y educación intercultural. Entre el neoindigenismo y la autonomía”, Revista Andamios. Volumen 3. Número 6, pp. 97-135.

FERNÁNDEZ, Julio y ARGÜELLO, Jaqueline (s/f), “Aspectos constitucionales del multiculturalismo en América Latina”, Pensamiento Constitucional. Año XVI. Número 16. ISSN 1027-676.

GOBIERNO FEDERAL MEXICANO (2014), Diario oficial. Miércoles 30 de abril de 2014. Décima Sección Extraordinaria. 
GOBIERNO FEDERAL MEXICANO (2014), Diario oficial. Lunes 14 de julio de 2014.

INEGI (2010), Censo poblacional.

KNIGHT, Alan (1995), “Continuidades históricas en los movimientos sociales”, Coord. Jane

Dale Lloyd y Laura Pérez Rosales Paisajes Rebeldes. Una larga noche de rebelión indígena. Universidad Iberoamericana. México.

LÓPEZ BÁRCENAS, Francisco, et al. (2001), “Los derechos indígenas y la reforma constitucional en México”, Serie Derechos Indígenas No. 1. Centro de Orientación y Asesoría a Pueblos Indios. México. LÓPEZ BÁRCENAS, Francisco (2004), "La lucha por la autonomía indígena en México: un reto al pluralismo", Coord. Aída Hernández, Sarela Paz y Teresa Sierra, El Estado y los indígenas en tiempos del PAN. Neoindigenismo, legalidad e identidad, Cámara de Diputados-CIESASPorrúa. México, pp. 207-331.

PÉREZ RUIZ, Maya Lorena (2005), ¡Todos somos zapatistas! Alianzas y rupturas entre el EZLN y las organizaciones indígenas en México. INAH. México.

PEQUEÑO, Andrea (2009), Participación y políticas de mujeres indígenas en contextos latinoamericanos recientes. FLACSO. Ecuador.

PIÑUEL RAIGADA, José Luis (2002), “Epistemología, metodología y técnicas del análisis de contenido", Estudios de Sociolingüística. 3(1): 1-42.

PIÑUEL RAIGADA, J.L. y GAITÁN, Moya, J.A. (1995), Metodología general. Conocimiento científico en investigación en la comunicación social. Editorial Síntesis. España.

RESTREPO, Eduardo (2011), "Etnización y multiculturalismo en el bajo Atrato", Revista Colombiana de Antropología. Instituto Colombiano de Antropología e Historia. Colombia.Vol. 47. Núm. 2.: 37-68.

REINA, Leticia (1980), Rebeliones campesinas en México (1819-1906). Siglo XXI. México.

SARMIENTO, Sergio y CONSUELO, Mejía (1988), La lucha indígena un reto a la ortodoxia. Siglo XXI. México.

STAVENHAGEN, Rodolfo (2002), "Identidad indígena y multiculturalidad en América Latina". Araucaria, Volumen 4. Número 7. Universidad de Sevilla. España.

VALLADARES, Laura (2001), “El debate indio sobre la ciudadanía multicultural en México: La revuelta por la dignidad", Centro de Investigaciones Etnológicas-Museo ArqueológicoUniversidad de los Andes. Boletín Antropológico 53: 313334, septiembre-diciembre.

VALLADARES, Laura (2008), "La política de la multiculturalidad en México y sus impactos en la movilización indígena: avances y desafíos en el nuevo milenio". Ed. Fernando García, Identidades, etnicidad y racismo en América Latina Colección 50 años FLACSO. Ecuador, pp. 289-308.

VELASCO, Ambrosio (2004), "Multiculturalismo, nación y federalismo”, Revista Mexicana de Ciencias Políticas y Sociales. Año/volumen XLVII. Número 191. UNAM. México, pp. 68-85.

\section{NOTAS}

1. Ver el caso de Chile en: Boccara y Boladis (2010), el caso de Colombia en: Restrepo (2011), y diversos estudios de nivel latinoamericano en: Pequeño (2009), Stavenhagen (2002) y Fernández y Argüello (s/f).

2. La Declaración de la ONU sobre los derechos de los pueblos indígenas fue aprobada por mayoría de la Asamblea General, en Nueva York, el 13 de septiembre de 2007. 
3. Pueden consultarse las políticas del Banco Mundial para la atención a los pueblos indígenas en: http://www.bancomundial.org/temas/resenas/indigenas.htm

4. Los procesos de movilización indígena en México tienen una larga historia que ha sido documentada desde la época colonial, por diversos autores como: Reina (1980), Sarmiento y Mejía (1988), Knight (1995), y Valladares (2008) registrando diversos períodos de protesta indígena que oscilan entre la rebelión y la negociación con el Estado mexicano.

5. Según el Diario Ofi cial, del Miércoles 30 de abril de 2014, en su Décima Sección-Extraordinaria.

6. De acuerdo con datos publicado en el Diario ofi cial en 30 de abril de 2014, en su décima sección extraordinaria.

7. INEGI, Censo poblacional 2010.

8. Según el registro ofi cial de la Cámara de Diputados disponible en: http:// www.diputados.gob.mx/ LeyesBiblio/ref/cpeum_crono.htm.

9. Ver el Diagnóstico presentado por la Comisión para los Pueblos Indígenas, en el Diario Oficial, del 30 de abril de 2014. Décima sección extraordinaria.

10. http://pulsoslp.com.mx/2014/10/31/gobierno-plantea-aumento-a-presupuesto-parapueblos-indigenas-en-0-47/

11. Aprobado por decreto presidencial del Gobierno de la República Mexicana, publicado en el Diario Oficial, el miércoles 30 de abril de 2014, en su Décima Sección Extraordinaria.

12. En México, la Secretaría de Gobernación es el equivalente a los Ministerios del Interior en otros países. Históricamente ha sido la encargada de reprimir los movimientos populares.

13. Aunque para facilitar la lectura se divide la información en 5 cuadros que concentran cada uno los datos de un sexenio en particular, el análisis diacrónico atraviesa en forma vertical a todos ellos. En este sentido, la lectura consecutiva de los cuadros 4, 5, 6, 7 y 8, permite visualizar fácilmente la línea del tiempo relacionada con los principales acontecimientos que han marcado la relación Estado Mexicano-población indígena, durante las últimas tres décadas, de una forma esquemática.

14. Existe sin embargo, una vasta producción de textos que abordan detalladamente la historia de los movimientos sociales en México. Una interesante historia que da cuenta, además del movimiento por los derechos de la población indígena, los movimientos agrarios en su lucha por la tierra, los movimientos sindicales y los movimientos estudiantiles; todos ellos con demandas específicas hacia el gobierno en turno, que van desde el reconocimiento de una identidad cultural, hasta la solución de problemas concretos; todos ellos respondiendo a ideologías propias, que coyunturalmente se han cruzado, sumando fuerzas, en lo que se conoce como la historia de la guerrilla mexicana. Un complejo entramado que, sin duda, merece ser objeto de un análisis posterior.

15. Fuentes testimoniales primarias.

16. Las críticas hacia los medios alineados al gobierno, en el conflicto reciente originado por La desaparición y muerte de 43 estudiantes normalistas en el Estado de Guerrero, han sido tema recurrente en la información presentada por medios internacionales, algunos medios nacionales independientes, y en los medios sociales en Internet, durante el último trimestre del 2014. 


\section{RESÚMENES}

En este trabajo se presenta un análisis de contenido del Programa Especial para los Pueblos Indígenas 2014-2018, del gobierno mexicano, a partir del modelo para la Mediación Dialéctica de la Comunicación Social (Piñuel, 2002), que permite trascender la simple identifi cación de datos de carácter intra-textual, hacia la descripción de las situaciones y el contexto implicados en la producción de dicho texto, como elementos determinantes de su contenido, con el objeto de formular un sistema conceptual explicativo de la relación actual del Estado mexicano con su población indígena.

Este trabalho apresenta uma análise do conteúdo do Programa Especial para los Pueblos Indígenas 2014-2018, do governo mexicano, a partir do modelo para a Mediación Dialéctica de la Comunicación Social (Piñuel, 2002), que permite transcender a simples identificação de dados de carácter intratextual e chegar à descrição das situações e dos contextos implicados na produção do referido texto como elementos determinantes de seu conteúdo, com o objetivo de formular um sistema conceptual explicativo da relação atual do Estado mexicano com a sua população indígena.

This paper presents a content analysis of the Mexican government Special Program for Indigenous People 2014-2018. The Dialectic Mediation of Social Communication Model (Piñuel, 2002) was applied, which allows to overcome a simple identification of data of intra-textual character to the description of the circumstances and context involved in the production of the text as determinants of its contents, in order to formulate an explanatory conceptual framework of the current relationship between Mexican state and its indigenous population.

\section{ÍNDICE}

Keywords: Multiculturalism, indigenous peoples, Mexican Government Palavras-chave: Multiculturalidade, povos indígenas, Estado mexicano Palabras claves: Multiculturalidad, Pueblos indígenas, Estado Mexicano

\section{AUTOR}

\section{ANDREA AGUILLAR EDWARDS}

Doctorada en Comunicación Social, pela Universidad de la Habana. Catedrática e Investigadora da Facultad de Ciencias de la Comunicación de la Universidad Autónoma de Coahuila. Miembro da Asociación Mexicana de Investigadores de la Comunicación (AMIC). Enderezo de correspondencia: Carretera a Zacatecas Km 2.5 Saltillo Coahuila 25000 México aguilaredwards@gmail.com 